\title{
El respeto de la autonomía como elemento fundamental en el tratamiento ético de los trastornos de la conducta alimentaria*
}

\author{
José Carlos Vázquez Parra ${ }^{a}$ Cassandra Michelle Martell Espericuetab
}

\begin{abstract}
Resumen: cuando se habla de trastornos de la conducta alimentaria, es usual pensar en las problemáticas médicas o psicológicas que implican este tipo de patologías. Sin embargo, no se puede negar que estas problemáticas vienen acompañadas de múltiples implicaciones sociales y personales por parte del individuo que se ve afectado, así como de los profesionales de la salud que participan en todo el proceso de atención. Por ende, el objetivo de este artículo es reflexionar sobre la necesidad de hacer un análisis ampliado de la realidad personal y social al momento de atender a pacientes con este tipo de trastornos. Para ello, es fundamental ver más allá de la simple patología médica y procurar que se respete siempre el principio de autonomía como piedra angular de la relación existente entre el médico y el paciente. Como conclusión, se busca que el profesional de la salud que interviene en procesos médicos relacionados con trastornos de la conducta alimentaria considere que la salud del paciente no solo consiste en superar el trastorno, sino también en el estado de bienestar durante el proceso terapéutico y sus implicaciones.
\end{abstract}

Palabras clave: psicopatología; bioética; deontología; ética médica

Recibido: 26 de noviembre de 2019 Aceptado: 26 de mayo de 2020

Disponible en línea: 15 de septiembre de 2020.

Cómo citar: Vázquez Parra JC, Martell Espericueta CM. El respeto de la autonomía como elemento fundamental en el tratamiento ético de los trastornos de la conducta alimentaria. Rev. latinoam. bioet [Internet]. 15 de septiembre de 2020]; 20(1). Disponible en: https://doi.org/10.18359/rlbi.4369

* Artículo de investigación producto del Verano de Investigación de la Academia Mexicana de Ciencias (AMC).

a Doctorado en Estudios Humanísticos, Maestría en Educación, Licenciatura en Derecho y Licenciatura en Psicología. Tecnológico de Monterrey, Campus Guadalajara. Zapopan, México.

Correo electrónico: jcvazquezp@tec.mx ORCID •

b Licenciatura en Psicología. Universidad de Guadalajara. Guadalajara, México.

Correo electrónico: michelle_martell_9630@hotmail.com 


\title{
Respect to autonomy as a key element in ethical treatment of eating disorders
}

\begin{abstract}
When talking about eating disorders, it is usual to think about medical or psychological problems this kind of pathologies imply. However, we can not deny this kind of problems come together with multiple social and personal implications regarding the affected individual, as well as the health professionals participating in the care process; hence, the objective of this article is to reflect upon the need for an extended analysis of the personal and social reality when treating patients with this kind of disorders. To do this, it is key to see beyond the simple medical pathology and try to always respect the autonomy principle as the cornerstone of the doctor-patient relationship. As a conclusion, it is sought that the health professionals intervening in medical processes related to eating disorders consider that the patient's health consists not only of overcoming the disorder, but also of the wellbeing state during the therapeutic process and its implications.
\end{abstract}

Keywords: psychopathology; bioethics; deontology; medical ethics

\section{O respeito da autonomia como elemento fundamental no tratamento ético dos transtornos do comportamento alimentar}

Resumo: Quando se fala de "transtornos do comportamento alimentar", é comum pensar nas problemáticas médicas ou psicológicas que esse tipo de patologias implica. Contudo, não se pode negar que essas problemáticas vêm acompanhadas de múltiplas implicações sociais e pessoas por parte do indivíduo que se vê afetado, bem como dos profissionais da saúde que participam de todo o processo de atenção. Nesse sentido, o objetivo deste artigo é refletir sobre a necessidade de analisar de forma ampla a realidade pessoal e social na atenção a pacientes com esse tipo de transtornos. Para isso, é fundamental ver mais além da simples patologia médica e procurar que seja respeitado sempre o princípio de autonomia como pedra angular do relacionamento existente entre médico e paciente. Como conclusão, espera-se que o profissional da saúde que intervém em processos médicos relacionados com transtornos do comportamento alimentar considere que a saúde do paciente não somente consiste em superar o transtorno, mas também no estado de bem-estar durante o processo terapêutico e suas consequências.

Palavras-chave: psicopatologia; bioética; deontologia; ética médica 


\section{Introducción}

A veces hablar de ética puede parecer un tema complejo para muchas personas, más aún cuando se trata de ética aplicada al ejercicio profesional. En disciplinas concretas como el área de la salud, hay constantes situaciones que ponen en entredicho la capacidad de tomar buenas y correctas decisiones, debido a que se genera un dilema entre las creencias, valores y principios de los agentes que prestan los servicios, así como de aquellos que los reciben (1).

No es necesario explicar detalladamente qué es la ética o profundizar en el tema, pues el objetivo de este artículo no es definir tales conceptos, sino abordar aspectos concretos sobre la ética aplicada a la presencia y tratamiento de los trastornos de la conducta alimentaria (TCA). Por consiguiente, basta con saber que la ética es una rama de la filosofía que reflexiona sobre la moralidad del comportamiento y evalúa el actuar a partir de principios que se consideran universalmente correctos para promover la vida en sociedad (2). Algunos de estos principios son: justicia, equidad, solidaridad, respeto a la vida, dignidad, integridad, honestidad, autonomía, verdad, entre otros (3). Estos principios deben ser respetados, pues son inherentes a toda persona $y$, por lo tanto, determinantes para promover el bien social(4).

La ética busca que el ejercicio profesional se adhiera a dichos principios con la intención de mejorar la relación entre los profesionales y sus clientes, así como con sus colegas, la profesión y la sociedad en general. Por consiguiente, la ética se configura como un pilar necesario para el ejercicio íntegro, responsable y sostenible de la profesión (5).

Según Cortina, citada por Ibarra (6), la profesión debe ser vista no solamente como una ocupación que permite obtener ingresos económicos y estatus social, sino también como una práctica social que adquiere su verdadero sentido y significado en el bien o servicio que proporciona a la sociedad. Aunado a esto, Cortina y Conill (7) consideran que todo profesional debe conocer los objetivos de su profesión, ya que, de no hacerlo, puede enfocarse en metas externas que desorientan su quehacer.

De ahí la necesidad de tener claridad sobre el objetivo de la profesión, ya que es una cuestión ética a la que los prestadores de servicios de salud deben enfrentarse con el fin de ayudar y velar por el bienestar del paciente, buscar siempre su beneficio (principio de beneficencia) y evitar en todo momento cualquier tipo de daño (principio de no maleficencia) (8).

Desde la ética profesional, el presente artículo busca hacer una aproximación a las acciones de los prestadores de servicios de salud, enfocándose en la atención de los TCA. Para desarrollar esta reflexión, se pretende abordar ciertas nociones básicas, así como el caso concreto de la alimentación asistida. Teniendo en cuenta el desarrollo de la perspectiva ética de estas patologías, se busca plantear un horizonte desde el cual se pueda construir una visión crítica sobre el rol que juega el principio de respeto a la autonomía de las personas.

\section{Nociones básicas}

\section{Alimentación}

La alimentación en los seres vivos es una necesidad básica y primordial. Según diversos autores, como el psicólogo humanista Abraham Maslow (9) o personajes como Manfred Max-Neef, Antonio Elizalde y Martin Hopenhayn (10), la alimentación es un aspecto de la vida humana que no solo le permite sobrevivir, sino que también, se configura como una manifestación social que se construye a la par del desarrollo.

Desde que están en el proceso de gestación, los seres humanos deben nutrirse para desarrollarse de manera óptima. Aunque en este primer momento el proceso de alimentación ocurre de forma pasiva, no se demerita el deseo instintivo de sobrevivir y la necesidad de alimentarse para llevarlo a cabo (11). Conforme pasa el tiempo, el ser humano comprende el binomio que existe entre la alimentación y el desarrollo, tomando consciencia de que, además de ser un proceso instintivo que responde a una necesidad, la alimentación es una conducta relacionada con hábitos y preferencias personales que se manifiestan a la par de lo que se come (12).

De acuerdo al conductismo radical, todo lo que el organismo realiza de manera consciente o 
inconsciente es considerado una conducta (13). Así mismo, clasificaremos el acto de alimentarse, pues requiere de la conciencia, sin que esto suponga un impedimento, como en el caso de la nutrición asistida.

Socialmente hablando, la alimentación implica el reconocimiento de factores que van más allá de la simple acción biológica de alimentarse, para incluir hábitos, preferencias y características regionales sobre qué se come, cuándo se come y cómo se come. No es extraño encontrar explicaciones sobre el rol que desempeña la comida y el acto de comer en las personas dependiendo del país en el que se localizan, en donde incluso se señalan aspectos rituales que se desarrollan a partir de la acción de comer. La comida forma parte de la historia, la religión y las costumbres de los grupos sociales, por lo que la conducta alimentaria no debe ser reducida a un simple acto nutricional.

Es importante señalar la complejidad del proceso de alimentación, pues como se dijo, de él hace parte no solo la necesidad biológica, sino también una serie de factores socioculturales y personales como los hábitos, los estilos de vida, las preferencias por ciertos alimentos e incluso, las dietas específicas de cada parte geográfica del mundo (14). De esta manera, se puede afirmar que el cuerpo humano responde a las necesidades instintivas, al deseo de ingerir cierto alimento y al aprendizaje de los patrones alimentarios.

Según Osorio, Weisstaub y Castillo (15), la conducta alimentaria es un comportamiento normal relacionado con los hábitos de alimentación, la selección de alimentos que se ingieren, las preparaciones culinarias y las cantidades ingeridas de ellos. Es decir, son aquellas conductas que cada persona tiene con los alimentos.

\section{Trastornos de la conducta alimentaria (TCA)}

Según la Guía de Práctica Clínica sobre Trastornos de la Conducta Alimentaria (16), estos se definen como el grupo de trastornos mentales caracterizados por la aparición de comportamientos de control de peso y conductas alteradas al ingerir alimentos. Esta alteración tiene como consecuencia problemas en el funcionamiento físico o psicosocial del individuo. La guía señala que estos trastornos se enfocan en el proceso de ingestión de alimentos, ya sea en cantidad o calidad, y no tanto en las consecuencias que pueden desencadenarse a partir de este desajuste de alimentación, como es el caso de la desnutrición, la anemia, la obesidad o el sobrepeso. Por otro lado, la guía no incluye en los trastornos a aquellas enfermedades que pueden causar que la conducta alimentaria se vea afectada, como la imposibilidad motora o la necesidad de dietas específicas que obliguen o inhabiliten la alimentación de un individuo (17).

En cuanto al diagnóstico de este tipo de trastornos, cabe señalar la existencia de diferentes clasificaciones que consideran aspectos complementarios y que, a su vez, contemplan diversos tipos de comportamientos alimentarios como parte de los trastornos de la conducta (18).

La Clasificación Internacional de Enfermedades (CIE) en su décima versión (19) incluye dentro de los TCA a: la anorexia nerviosa, la anorexia nerviosa atípica, la bulimia nerviosa, la bulimia nerviosa atípica, la hiperfagia asociada a otros trastornos psicológicos, los vómitos asociados a otros trastornos psicológicos, así como otros trastornos de la conducta alimentaria y el trastorno de la conducta alimentaria sin especificación.

Por su parte, el Manual Diagnóstico y Estadístico de los Trastornos Mentales (MDE) en su quinta versión 20), incluye a la pica, la rumiación, los trastornos de la evitación/restricción de alimentos, la anorexia nerviosa, la bulimia nerviosa, el trastorno de atracones, y otros trastornos de la conducta alimentaria o de la ingesta de alimentos especificados y no especificados.

De manera adicional, algunos otros trastornos no incluidos en estas clasificaciones, que también afectan la conducta alimentaria, son: la ortorexia, la diabulimia, la ebriorexia, la permarexia, la megarexia o incluso, la vigorexia, si esta altera o modifica la alimentación del individuo (21).

La variedad de trastornos ocasiona que en muchas circunstancias las personas no cuenten con un diagnóstico exacto sobre lo que les afecta directamente, pues a veces los criterios no se cumplen por la temporalidad o latencia en la que se manifiestan 
las conductas o porque no se expresa toda la sintomatología, dando como resultado que muchos de los casos sean diagnosticados como trastornos no especificados (22).

No obstante, aunque no se cuente con un diagnóstico certero, la intervención debe considerarse urgente, debido a que sin un tratamiento adecuado se pone en riesgo la salud y la vida del paciente (23). Por esto se considera importante que el profesional de la salud identifique la presencia de este tipo de anomalías del comportamiento.

Estas cuestiones resultan fundamentales para comprender la ética del profesional de la salud y su relación con los TCA, ya que este tipo de fenómenos se enmarcan dentro de una realidad compleja con variadas aristas que deben ser consideradas para poder concluir si el proceder de cierto profesional es correcto o incorrecto.

\section{TCA: impacto social y de salud pública}

Sin lugar a dudas, el pensamiento y los valores compartidos por la sociedad han cambiando a lo largo de los años. Los ideales de belleza y salud del cuerpo, así como la imagen que se tiene de él, configuran un patrón de comportamiento relacionado con la alimentación, el ejercicio, la nutrición y el desarrollo (24). De esta manera, se puede señalar que la concepción de los estilos de vida considerados saludables corresponden al desarrollo de la sociedad en la que están inmersos.

Aunque una primera reflexión sobre los TCA sugiera que su manifestación es relativamente reciente, según Baile y González (17), es posible que estos comportamientos existan desde tiempo atrás, pero presentados de diversas maneras, así como asociados a otros factores socioculturales.

Cuando se habla de algún trastorno en el área clínica, es importante resaltar los análisis explicativos de los factores predisponentes relacionados con la conducta atípica (25). En el caso de estos trastornos hay elementos que pueden ser desencadenantes de conductas de alimentación clínicamente clasificables (17), como: el género, ciertos tipos de personalidad, el concepto que se tiene de sí mismo y los demás o las presiones sociales.
$\mathrm{Al}$ identificar los factores, se puede presumir una posible relación de estos trastornos con el entorno social del paciente (26).

Si bien estos trastornos no son exclusivos de la contemporaneidad, en los últimos 50 años su presencia ha aumentado del 1 al 3\% en mujeres jóvenes. Este cambio ha llamado la atención y se ha declarado como un problema de salud pública en el mencionado grupo poblacional (27). Según la Agencia para la Investigación y Calidad en el Cuidado de la Salud de Estados Unidos (AHRQ, por su sigla en inglés), entre 1999 y el 2006 (28) las hospitalizaciones causadas por trastornos de la conducta alimentaria se elevaron de manera abrupta en un $18 \%$, y afectaron alrededor de 70 millones de personas en el 2016 (29).

Así, resulta importante reflexionar sobre la manera en que estos tipos de trastornos suelen afectar en una proporción de 1:10 a las mujeres, en comparación a los hombres (22). Álvarez, Franco, López, Mancilla y Vázquez (30) mencionan que en México, tras un estudio realizado a mujeres de 25 a 45 años, se encontró que el 79 \% reportó insatisfacción con su cuerpo, el 70 \% deseaba adelgazar, aun cuando estaba en su peso. Por otro lado, se identificó que las que decían estar satisfechas tenían un peso bajo.

Pese a que en algunas investigaciones los trastornos se presentan con mayor tendencia en el género femenino, la prevalencia en los últimos años se ha ido modificando (31). En un estudio realizado en Estados Unidos por Hudson, Hiripi, Pope y Kessler (32), aproximadamente veinte millones de mujeres y diez millones de hombres han presentado algún trastorno de la conducta alimentaria en su vida.

Otra asunto importante es la percepción social construida en torno a este tipo de trastornos y a las personas que los padecen. A pesar de ser una cuestión de salud, gracias a la influencia de los medios de comunicación en ocasiones los TCA se califican de manera errónea al considerarlos comportamientos a los que incurren los jóvenes para llamar la atención o para ser parte de una moda (33). Lamentablemente, estas falsas creencias sobre los trastornos de la conducta alimentaria restan importancia al tema, desenfocan la atención y 
afectan la posibilidad de tener ideas correctas sobre la situación real (34).

En relación con la influencia de los medios de comunicación se destaca el caso ocurrido durante la semana de la moda de Nueva York del 2017, en donde varias marcas de moda hicieron el compromiso de no contratar a modelos extremadamente delgadas o menores de 16 años (35), con el fin de contrarrestar en los jóvenes la influencia social que suele tener la imagen de las modelos. Sin embargo, el acuerdo no incluyó modificaciones en los parámetros de tallas o publicidad externa (36), pero sí subrayó la gravedad de la presencia de trastornos en la comunidad que se dedica a la moda, lo que resultó, según Losada, Leonardelli y Magliola (36), en un mensaje contraproducente.

De esta manera, la información errónea o sesgada del problema da pauta a una serie de estereotipos que desencadenan juicios de valor hacia las personas que padecen estos trastornos. Los adjetivos basados en los desórdenes, como la anorexia o la bulimia, etiquetan a los individuos y los segregan de su grupo social (37). Así, la cosificación de una persona con un TCA expone el padecimiento del individuo, atenta contra su dignidad, violenta y cuestiona cualquier acción que pueda llevarse a cabo para ayudarla.

De lo anterior se puede afirmar que la atención de los trastornos de la conducta alimentaria se enfrenta a múltiples variables que trascienden el terreno de la salud, lo que tiene fuertes implicaciones que recaen sobre los responsables de la atención de este tipo de pacientes (38). Si los profesionales no consideran todos estos factores y elementos que se construyen en torno del problema, el tratamiento que se lleve a cabo puede terminar siendo un paliativo, poco efectivo o, incluso, contrario al bienestar del paciente.

\section{Implicaciones éticas de los TCA en el actuar de los profesionales de la salud}

El manejo integral en los TCA es algo esencial y fundamental para el tratamiento y proceso de recuperación de los pacientes, por lo que una visión demasiado acotada del problema implica no considerar un factor que detone una nueva presencia del trastorno a corto, mediano o largo plazo (39).

Cabe reiterar que la responsabilidad del profesional de la salud implica la necesidad de reconocer en el paciente a una persona que no solo le refleja un trastorno en su conducta, sino que también tiene una vida y se desempeña en un grupo social determinado. Por tanto, el paciente es un agente autónomo y requiere ser tratado con dignidad (40). Considerando las implicaciones de los TCA, es necesario que la atención se geste dentro de un marco ético en el que los valores y principios del profesional y del paciente sean considerados y respetados por igual.

Según Siurana (41), la ética de un profesional de la salud conlleva de manera natural la necesidad de considerar principios de bioética para cualquier decisión sobre la atención, tratamiento y recuperación del paciente, teniendo en cuenta las implicaciones éticas, sociales, legales, filosóficas y de creencias que surgen del cuidado de la salud y la relación médico-paciente.

A diferencia de la prestación de un servicio que puede darse de manera unilateral, la atención médica requiere de la constitución de una relación entre el profesional de la salud y el paciente que dé cabida a un intercambio de creencias sobre lo que correcto, importante y valioso (42).

De manera concreta, los TCA son un reto para su atención por las implicaciones sociales y los juicios de valor que respaldan o condenan estos comportamientos, los cuales pueden desencadenar en demandas legítimas o ilegítimas por parte de los pacientes hacia el profesional que los atiende (43). Por lo tanto, la atención se debe respaldar en marcos de actuación ética porque, al basarse en principios universales, subsanan muchos de los juicios de valor que se sustentan en perspectivas morales, enfoques legales o visiones de corte social, emocional o incluso religioso (44).

Ahora bien, al hablar de bioética en los aspectos de salud se debe reconocer que la cuestión biológica no es la única que define la vida de un ser humano. Por su complejidad, existen múltiples implicaciones no biológicas que se relacionan con la vida humana, como los intereses, manera de vivir, 
creencias, motivaciones y deseos (45). Según la Organización Mundial de la Salud (OMs) (46), la salud debe percibirse más allá de la ausencia de enfermedades y dolor y, en cambio, centrarse en un estado de bienestar. Entonces, cabe preguntarse si el objetivo de un profesional de la salud consiste en recuperar la salud física, salvar la vida o, en cambio, conseguir el bienestar de sus pacientes. De acuerdo a la perspectiva del presente texto, las tres opciones son parte de un mismo objetivo que debe garantizarse. Sin embargo, el punto central de este artículo reside en el enfoque del bienestar y, desde la bioética, de la responsabilidad de todo profesional de la salud. En el caso de los TCA, existe una mayor necesidad de salvaguardar y de considerar las implicaciones que trae consigo el tratamiento de estos trastornos. La práctica médica de atención de los TCA no debe únicamente valorar y salvaguardar la vida, sino también velar por el bienestar y el respecto de la dignidad de los pacientes, considerándolos en todo momento como seres autónomos y responsables de sus propias decisiones.

\section{La ética de la práctica médica}

Aunque el término bioética es una noción que surge hacia la década de 1970 en los Estados Unidos, la ética del profesional de la salud se relaciona más con la idea del ethos profesional, es decir, el uso o valores que enmarcaban el actuar de estos especialistas en las diferentes etapas de la historia (47). Desde el siglo V a.C., el Juramento de Hipócrates señalaba distintos principios que modelan hoy en día la práctica médica y que se pueden visualizar en diversos códigos deontológicos y declaraciones internacionales, como los Códigos de la Asociación Médica Mundial (AMM) o las Declaraciones de Helsinki, Tokio, Venecia, Hong Kong y Lisboa sobre la práctica médica y los derechos del paciente (48).

Sin embargo, un momento determinante para la ética de la práctica médica fueron los principios clásicos de la medicina con la Declaración Universal de los Derechos Humanos (DUDH), los cuales obligaban a que el profesional considerara a su paciente con la misma dignidad y respeto que tendría consigo mismo (49). Así, se da una convergencia entre la ética médica y los derechos que protegen a todo ser humano. Esto luego permite que en la década de 1960 se dé una reivindicación social de las partes del ejercicio profesional y se afirmen los derechos individuales dentro de la práctica médica. Esta situación denunció el paternalismo de los médicos en la relación terapéutica y la falta de respeto a los sujetos en algunas de sus prácticas en las que se imponía el modelo técnico científico sobre el paciente (50).

Conforme pasaron los años, la relación entre ética y medicina se enfocó primeramente en recuperar el equilibrio en la relación médico-paciente. No obstante, se ha evidenciado una alarmante deshumanización de las personas ante el progreso de la medicina en temas tecnológicos, a causa de la involuntaria cosificación que se ha dado del paciente. Algunos autores cono Kraus y Cabral (51) consideran que la medicina perdió sus dimensiones humanas cuando olvidó el carácter meramente instrumental de la tecnología e hizo de esta un fin por sí mismo.

De esta manera, las relaciones entre el médico $y$ el paciente pueden tener diferentes implicaciones y alcanzar distintos niveles, ya que en algunos países el paciente sigue aceptando unilateralmente lo que el médico le señale con respecto a su salud; mientras que en otras regiones la relación se da de una forma mucho más bilateral y de respeto mutuo (52). Cabe señalar que en algunos casos los médicos deben tomar ciertas decisiones de manera unilateral, dependiendo del caso del paciente; sin embargo, en la mayoría prima el respeto a la autonomía del paciente y lo que enaltezca la relación profesional entre médico y paciente (50).

Aunque se podrían mencionar los principios de beneficencia, no maleficencia, protección de la vida, integridad, entre otros, para los fines de este artículo se hará énfasis en el de respeto a la autonomía, pues este principio pone en juego los TCA, por los conflictos entre los intereses del paciente y el médico que pueden suscitarse al momento de un tratamiento.

\section{Principio de respeto a la autonomía}

El principio de autonomía hace referencia a la libertad que tiene un individuo para elegir y actuar con base en sus creencias e ideales, sin verse influido 
u obligado por otro. La autonomía significa saber pensar y actuar por sí mismo; en otras palabras, es una capacidad para tomar decisiones y gestionar a la propia persona y el propio cuerpo (53).

En el terreno de la práctica médica, la autonomía tiene relación con el respeto de esta libertad del paciente, de decidir sobre cualquier proceso al que pueda estar sujeto, sin que el profesional de la salud se anteponga, considerando que debe respetar al paciente y considerarlo como un agente con capacidad de elección y acción (54). Teniendo en cuenta que el paciente no es un objeto manipulable por parte de los especialistas, el ejercicio de la medicina implica el respeto a la relación terapéutica en la que tanto profesional como paciente participen como agentes independientes (55).

Entonces, este principio ético postula el respeto a las decisiones autónomas del paciente; por tanto, el médico tendría un papel de facilitador. Sin embargo, cabe señalar que existen límites a las peticiones o decisiones de todo paciente, pues el respeto a la autonomía no es un principio absoluto (55). Así, aunque los principios del paciente son importantes, lo son de igual manera los del personal de salud y dan cabida a posibles situaciones en que se contrapongan las visiones y creencias de ambas partes de la relación profesional (44).

Considerando la posibilidad de estas situaciones dilemáticas de conflicto de intereses entre paciente y profesional, la autonomía debe responder a parámetros mínimos de consciencia que no afecten el estado de libertad del individuo. Si a causa de algún elemento externo o factor médico el paciente carece de las características mínimas que garanticen su libertad o consciencia, la autonomía de sus decisiones puede llegar a ser cuestionada (53). Sin embargo, se comprende que la consciencia no es un parámetro de valoración completamente confiable, ya que en el terreno médico es común que el paciente esté condicionado por su propia enfermedad o factores relacionados (dolor, miedo, desconocimiento). Esto también puede cuestionar su verdadero estado de autonomía en la toma de decisiones (50). Por ende, el profesional de la salud se enfrenta a difíciles dilemas al momento de querer respetar el principio de autonomía, pues apegarse únicamente a las decisiones del paciente no es una elección correcta cuando el padecimiento afecta el estado mental del individuo.

En casos como los TCA, analizar los casos concretos marca una significativa diferencia al momento de tomar una decisión sobre el abordaje médico de un paciente. Con esto se busca evitar la presencia de dilemas que confronten al paciente $\mathrm{y}$ al profesional sobre el futuro de su tratamiento. Para aclarar este punto, se presenta el caso del soporte alimentario nutricional.

\section{El principio de autonomía ante los tratamientos de soporte alimentario nutricional en pacientes con TCA}

Con soporte nutricional se hace referencia a aquel tratamiento que debe llevarse a cabo cuando una persona no puede alimentarse por voluntad propia. En este proceso se encuentran involucrados múltiples profesionales de la salud, los cuales pueden participar desde la atención en la sintomatología, hasta en las afectaciones por llevar a cabo este tipo de intervención médica (56). Este es un proceso que incumbe a médicos, nutriólogos, personal de enfermería y atención hospitalaria.

El uso de este tipo de atenciones nutricionales es muy usual en pacientes que, a causa de padecimientos que imposibilitan su movilidad o afectan su consciencia, requieren del apoyo de un profesional de la salud para nutrirse o hidratarse. Esta atención se lleva a cabo desde la conformidad del paciente o familiares, con el fin de respetar su deseo de ser apoyado (42).

Sin embargo, hay casos en que no se cuenta con un impedimento por parte del paciente para alimentarse o la voluntad expresa para permitir ser alimentado, lo que da cabida a dilemas éticos que contraponen la búsqueda del bienestar y salud del paciente. No es extraño encontrar pacientes con TCA muy desarrollados que se niegan a ser alimentados o hidratados por los profesionales de la salud; en estos casos ejercen su libertad de decisión por encima de su propia vida (39).

Para muchos académicos, la autonomía del paciente puede darse por alto cuando se trata de un cuidado de preservación de la vida (57); en estos 
casos prima la responsabilidad de los profesionales de la salud que le atienden (58). En este sentido, Collazo (44) considera que cuando peligra la vida del paciente, será elección del médico si pone o no a consideración del individuo la decisión de llevar a cabo la alimentación asistida como parte de una intervención médica, y debe informarle al paciente que de ello depende el proceso inicial. Por lo anterior, es importante que los profesionales de la salud diferencien los casos en que la hidratación o alimentación asistida es necesaria para la preservación de aquellas situaciones en las que se busca mejorar la calidad de vida (44).

Con los TCA puede existir un deseo expreso por no alimentarse o no hacerlo de la manera adecuada. En estos casos, no es posible simplemente obligar a la persona, ya que esto violentaría su autonomía (23). Sin embargo, si a causa de estos trastornos se deriva una situación agravada de salud o daño en sus órganos que ponga en riesgo su vida, será obligación del equipo de profesionales de la salud utilizar un tratamiento de soporte alimentario nutricional, únicamente con el fin de conservar al paciente con vida. Una vez se consiga estabilizar al paciente, se debe buscar su consentimiento para llevar a cabo otros procedimientos médicos (8).

En los casos en que no haya conciencia por parte del paciente, o su estado esté muy deteriorado, se puede buscar el apoyo de la familia para tomar algunas de las decisiones en conjunto. Sin embargo, una vez la persona se recupere, se le deberá considerar en las decisiones sobre su salud. Como parte del respeto al principio de autonomía, el paciente debe ser partícipe de su tratamiento, conocer todas las variables de la decisión y estar bien orientado sobre las opciones que tiene ante la intervención médica (49). Por este motivo, Moreno y Galiano (59) consideran necesario que el personal de salud desarrolle una relación de confianza con el paciente y le otorgue la información que pudiera necesitar para tomar la mejor decisión sobre su tratamiento.

Los TCA suelen venir acompañados de creencias y factores sociales que alteran la visión del paciente acerca de su situación real o de las implicaciones de determinado tratamiento; por tanto, es importante que el equipo médico comprenda que el proceso de alimentación desempeña un rol conflictivo en estos casos y que realizarlo o no puede generar un problema con el paciente pues, aunque mejore su salud, no necesariamente beneficia su estado de bienestar (53).

Una situación distinta que se debe tener en cuenta es cuando la persona se encuentra en coma o en un estado vegetativo y la familia es la encargada de tomar las decisiones. En estos casos, es importante verificar si existen manifestaciones textuales de la voluntad de las personas acerca de los cuidados y tratamientos que hubiera querido recibir en una situación así. Esto permite considerar la autonomía del individuo, aunque en dicho momento no pueda externar su padecer (50).

Desde un enfoque ético de respeto al principio de autonomía, es importante revisar el estado de la persona y preguntarse: ¿se está luchando por mantener con vida al paciente al nutrirlo?, ¿cómo su calidad de vida se ve afectada utilizando intervenciones para alimentarse? o ¿acaso intentar alimentarla es más perjudicial? (44). Así, la alimentación artificial se suministra para el mantenimiento de la vida cuando hay enfermedades graves, sean reversibles o no, así como cuando el paciente ha perdido la capacidad para razonar las ventajas de la alimentación (54). Cabe aclarar que si la ausencia de nutrición e hidratación provocan en el paciente la aceleración o causa de muerte, no es ético suspender la alimentación (60). Además, se deben considerar los casos en donde se pueda suspender el proceso de nutrición asistida por ser irrelevante para el estado de salud del paciente; asimismo, considerar todas las variables del caso concreto y tratar de hacer lo posible por no afectar al paciente y permitirle un buen morir de manera digna (61).

Sobre este último punto es importante recordar que es una obligación, al igual que el respeto a la autonomía del paciente, que el profesional de la salud no incurra en actos que afecten al paciente (principio de no maleficencia). Por lo tanto, aunque el acto de alimentarse no es perjudicial por sí mismo, es posible que algunos métodos afecten de manera negativa, razón por la cual debe realizarse un análisis mayor de la situación del paciente (54).

Como se puede apreciar en este caso concreto, el respeto al principio de la autonomía, así como el apego a otros principios de bioética en los que 
participan los pacientes o profesionales de la salud, requieren una valoración amplia y profunda por parte del agente decisor. Este debe atender las necesidades médicas que la situación requiere en cierto momento, así como considerar todas las implicaciones que puede tener su actuación en la vida y el futuro del paciente, con el fin de responder más allá de lo relacionado con su salud.

De lo anterior se puede afirmar que la presencia de los TCA, trae consigo implicaciones que trascienden la respuesta médica, lo cual implica variadas situaciones que se relacionan con la vida, el entorno social, las creencias y los deseos de la persona, quien, a pesar de su trastorno, debe ser respetada. La despersonalización del paciente con un TCA no solo atenta contra la ética médica, sino que además, desensibiliza al profesional de su obligación primordial de cuidar la vida y buscar el bienestar de las personas.

\section{Consideraciones finales}

Hoy en día la visión y los objetivos del perfil médico han cambiado. Un profesional de la salud no solo previene y atiende enfermedades y lesiones, $o$ asiste en la curación y el cuidado de los enfermos, sino que también vela por la promoción y mantenimiento de la salud y por el mejoramiento de la calidad de vida y bienestar de los pacientes; con el propósito de salvaguardar, en todo momento, la dignidad y el respeto de su individualidad (41).

En el caso de los TCA, otros profesionales de la salud como los psiquiatras, los psicólogos y los nutriólogos, desempeñan un rol determinante en la atención y las decisiones que se toman en los procesos de recuperación de los pacientes y, al igual que los médicos, deben atenerse a las prácticas éticas y responsables de su profesión. En cuanto al enfoque ético de esta problemática, es importante subrayar la relevancia de contar con un equipo de atención multidisciplinar que permita atender de manera integral al paciente, lo cual puede hacer la diferencia entre la adopción o rechazo de un tratamiento de alimentación asistida o artificial. Es posible que al final de una buena explicación al paciente el resultado no sea el esperado; sin embargo, el hecho de brindar opciones, respetar la libertad y considerar la autonomía del paciente es lo que debe procurar un equipo médico.

Así, es menester que los profesionales de la salud revaloricen la razón de ser de su profesión, para hacer consciente el enfoque de su labor e identificar si únicamente esta centrado en el mantenimiento de la vida, o si además tiene en cuenta la conservación de la salud, la dignidad y el bienestar de sus pacientes. Este punto resulta fundamental, al momento de tratar los TCA.

\section{Referencias bibliográficas}

1. López M. Ética profesional y complejidad. Perfiles Educativos. 2013; xxxv (142), 43-53.

2. Cortina A. Ciudadanos del Mundo. Hacia una teoría de la ciudadanía. 3ra ed. Ética de las profesiones. Madrid: Alianza; 1997. Ética de las profesiones; p. 146-163.

3. Del Río Sánchez C. Guía de ética profesional en psicología clínica. Ciudad de México: Grupo Anaya; 2018.

4. Alcántara A., Barba L., Hirsch A. Valores universitarios y profesionales de los estudiantes de posgrado de la unam. Ciudad de México: unam; 2009. Valores de ética profesional. p. 181-242.

5. Escobar B, Cid P. El cuidado de enfermería y la ética derivados del avance tecnológico en salud. Acta bioeth. [Internet]. 2018; 24(1): 39-46. Disponible en: http:// dx.doi.org/10.4067/S1726-569X2018000100039.

6. Ibarra G. Ética y valores profesionales. REencuentro. Análisis de Problemas Universitarios [Internet]. 2007; (49): 43-50. Disponible en: https://www.redalyc.org/ articulo.oa?id=34004907.

7. Cortina A, Conill J. 10 Palabras Clave en Ética de las Profesiones. Navarra: Verbo Divino; 2000.

8. Milos P. Ejercicio profesional y responsabilidad de la enfermera. Rev. Horizonte de Enfermería. 2019; 8(2): 12-19.

9. Maslow A. A theory of human motivation. Psychol. Rev [Internet] 1943;50(4):370-396. Available from: https://doi.org/10.1037/h0054346

10. Max-Neef M, Elizalde A, Hopenhayn M. Desarrollo a escala humana: conceptos aplicaciones y algunas reflexiones. Santiago de Chile: Cepaur; 1986.

11. Gómez L. Conducta alimentaria, hábitos alimentarios y puericultura de la alimentación. Precop SCP [Internet]. 2008;7(4):38-50. Disponible en: https://issuu. com/precopscp/docs/precopvol7n4_5

12. Forero T. Y, Acevedo R. M, Hernández M. J, Morales S. G. La alimentación complementaria: 
Una práctica entre dos saberes. Rev. Chil. Pediatr [Internet]. 2018;89(5): 612-620. Disponible en: https://doi.org/10.4067/S0370-41062018005000707

13. Ramnerö J, Törneke N. The ABCs of human behavior: Behavioral principles for the practicing clinician. Estados Unidos: New Harbinger Publications; 2008.

14. Tormo M, Trescastro E, Galiana M, Pascual M, Bernabeu J. Malnutrición y desigualdades en la España del desarrollismo: las encuestas rurales de alimentación y nutrición. Nutr. Hosp [Internet]. 2018, 35(№ Extra 5): 116-122. Disponible en: doi:10.20960/nh.2093

15. Osorio J, Weisstaub G, Castillo C. Desarrollo de la conducta alimentaria en la infancia y sus alteraciones. Rev. chil. nutr [Internet]. 2002;29:280-285. Disponible en: http://dx.doi.org/10.4067/S0717-75182002000300002 .

16. Ministerio de Sanidad y Consumo. Guía de Práctica Clínica sobre Trastornos de la Conducta Alimentaria. Barcelona: Generalitat de Cataluña. Departamet de Salut; 2009.

17. Baile A, González M. Trastornos de la Conducta Alimentaria antes del Siglo xx. Psicología Iberoamericana. RIP [Internet]. 2010; 18(2): 16 -26. Disponible en: https://www.redalyc.org/pdf/1339/133915921003.pdf

18. Figueroa G. Bioética de la anorexia nerviosa ¿autonomía, beneficencia o responsabilidad? Rev. Méd. Chile [Internet]. 2008; 136(5): 659-665. http://dx.doi. org/10.4067/S0034-98872008000500017.

19. Organización Mundial de la Salud (oMs). CIE 10. Décima revisión de la Clasificación Internacional de Enfermedades. Trastornos mentales y del comportamiento. Madrid: Meditor Editorial; 1992.

20. American Psychological Association (APA). Diagnostic and Statistical Manual of mental Disorders. Washington: APA; 2013.

21. Méndez J, Vázquez V, García E. Los trastornos de la conducta alimentaria. Bol. Med. Hosp. Infant. Mex [Internet]. 2008; 65(6): 579-592. Disponible en: https:// www.medigraphic.com/pdfs/bmhim/hi-2008/hi086n. pdf

22. López C, Treasure J. Trastornos de la Conducta Alimentaria en adolescentes: Descripción y manejo. RMCLC [Internet] 2010; 1(22): 85-97. Disponible en: DOI: $10.1016 / \mathrm{S} 0716-8640(11) 70396-0$

23. Buso C. Trastornos de la conducta alimentaria. Rev. Información psicológica. 2018; (116), 1.

24. Maganto C, Cruz S. La imagen corporal y los trastornos alimenticios: una cuestión de género. Sepypna [Internet]. 2000; (30): 45-48. Disponible en: https://www. sepypna.com/documentos/articulos/maganto-imagen-corporal-trastornos-alimenticios.pdf
25. Sáenz S, González F, Díaz S. Hábitos y trastornos alimenticios asociados a factores socio-demográficos, físicos y conductuales en universitarios de Cartagena, Colombia. Rev. Clin. Med. Fam [Internet]. 2011; 4(3): 193-204. Disponible en: http://dx.doi.org/10.4321/ S1699-695X2011000300003

26. Moreno J. Moral corporal, trastornos alimentarios y clase social. Madrid: Centro de Investigaciones Soiológicas; 2010.

27. Vázquez R, López X, Ocampo M, Mancilla J. El diagnóstico de los trastornos alimentarios del DSM-IV-TR al DSM-5. Rev. Mex. de trastor. Aliment [Internet]. 2015; 6(2); 108-120. Disponible en: http://dx.doi.org/10.1016/j.rmta.2015.10.003

28. Agency for Healthcare Research and Quality (AHRQ) 2018. Disponible en U.S. Department of Health and Human Services: https://www.ahrq.gov/topics/informacion-en-espanol/index.html

29. Arguello L. Arellanes A. Padecen 70 millones de personas algún trastorno alimenticio. Mujeres por Sinaloa; 2017 jun. 02. Disponible en: https://mujeresporsinaloa.com/2017/06/02/padecen-70-millones-de-personas-algun-trastorno-alimenticio/

30. Álvarez-Rayón G, Franco-Paredes K, López-Aguilar X, Mancilla-Díaz J, \& Vázquez-Árevalo R. Imagen corporal y trastornos de la conducta alimentaria. Rev. salud pública [Internet]. 2009; 11(4): 568-578. Disponible en: https://doi.org/10.1590/S0124-00642009000400008

31. Escolar MC, Martínez MÁ, González MY, Medina M, Mercado E, Lara, F. Factores de riesgo de trastornos de la conducta alimentaria entre universitarios: Estimación de vulnerabilidad por sexo y edad. Rev. Mex. de trastor. Aliment [Internet]. 2019; 8(2): 105-112. Disponible en: http://www.scielo.org.mx/scielo.php?scrip$\mathrm{t}=$ sci_arttext\&pid=S2007-15232017000200105

32. Hudson J, Hiripi E, Pope H, Kessler R. The prevalence and correlates of eating disorders in the National Comorbidity Survey Replication. Biol. Psychiatry. 2007 febr. 1; 61(3): 348-358. Disponible en: DoI: 10.1016/j. biopsych.2006.03.040

33. Gómez C, Palma S, Miján A, Rodríguez P, Matía P, Loria V, Martín Á. Consenso sobre la evaluación y el tratamiento nutricional de los trastornos de la conducta alimentaria: anorexia nerviosa. Nutr. Hosp [Internet]. 2018; 35(1): 11-48. Disponible en: DoI: 10.20960/ nh.1561

34. Castelao O, Blanco A, Meseguer C, Thuissard IJ, Cerdá B, Larrosa M. Estilo de vida y riesgo de trastorno alimentario atípico en estudiantes universitarios: realidad versus percepción. Enfermería Clínica. 2019; 29(5): 280-290. 
35. Infobae. Dos grandes firmas de moda le dicen basta a la promoción de la anorexia. 2017 sept. 07. Disponible en: https://www.infobae.com/tendencias/ lifestyle/2017/09/07/dos-grandes-firmas-de-moda-ledicen-basta-a-la-promocion-de-la-anorexia/

36. El observador. Importantes marcas prohíben modelos demasiado delgadas y menores de 16. 2017 sept. 06. Disponible en: https://www.elobservador.com.uy/importantes-marcas-prohiben-modelos-demasiado-delgadas-y-menores-16-n1115418

37. Losada A, Leonardelli E, Magliola M. Influencia sociocultural y los trastornos de la conducta alimentaria en adolescentes. Rev. Elec. Psic Izt [Internet]. 2017; 18(1): 381-416. Disponible en: https://www.medigraphic. com/pdfs/epsicologia/epi-2015/epi151s.pdf

38. Nuñez A. Trastornos de la alimentación: un verdadero problema en adolescentes. Rev. Hisp. Cienc. Salud [Internet]. 2016; 2(1): 110-111. Disponible en: http://www. uhsalud.com/index.php/revhispano/article/view/125

39. Morales JL. Análisis del comportamiento alimentario y sus factores psicológicos en población universitaria no obesa. Anales de Psicología [Internet]. 2018; 34(1): 1-6. Disponible en: http://dx.doi.org/10.6018/analesps.34.1.294341

40. Slachevsky C, Abusleme L, Teresa M, Arenas A. Cuidados paliativos en personas con demencia severa: reflexiones y desafíos. Rev. méd. Chile [Internet]. 2016; 144(1): 94-101. Disponible en: http://dx.doi. org/10.4067/S0034-98872016000100012

41. Siurana JC. Los principios de la bioética y el surgimiento de una bioética intercultural. Veritas [Internet]. 2010; 22: 121-157. Disponible en: http://dx.doi. org/10.4067/S0718-92732010000100006

42. Urrutia M, Cazenave A, Badillo H, Fajardo O. Trastornos de la conducta alimentaria en adolescentes. Rev. Horizonte de Enfermería. 2019; 19(1): 13-21.

43. Pardo A. La ética en la medicina. Persona y bioética [Internet]. 2011; 15(2): 166-183. Disponible en: https:// doi.org/10.5294/pebi.2011.15.2.4

44. Collazo E, Girela E. Problemas éticos en relación a la nutrición y a la hidratación: aspectos básicos. Nutr. Hosp. [Internet]. 2011; 26(6): 1231-1235. Disponible en: http://scielo.isciii.es/scielo.php?script=sci_arttext\&pi$\mathrm{d}=\mathrm{S} 0212-16112011000600006$

45. Capdevila, J. Novedades en Trastornos de la conducta alimentaria. Información psicológica. 2018; (116): 138-139.
46. Alcántara G. La definición de salud de la Organización Mundial de la Salud y la interdisciplinariedad. Sapiens [Internet]. 2008; 9(1): 93-107. Disponible en: https:// www.redalyc.org/pdf/410/41011135004.pdf

47. Pfeiffer M. La bioética como brecha. El Ágora [Internet]. 2013; 13(1): 1-3. Disponible en: https://doi. org/10.21500/16578031.102

48 Valls R. Ética para Bioética. Rev. de Bioética y Derecho [Internet]. 2011; (23): 1-27. Disponible en: https://www. redalyc.org/pdf/783/78339725005.pdf

49. Mendieta G, Cuevas J. Bioética de la salud pública. Rev. Latinoam. Bioet [Internet]. 2017; 17(2): 8-17. Disponible en: https://doi.org/10.18359/rlbi.2929

50. Hernández L. Relación médico-paciente y la calidad de la atención médica. Revista Conamed [Internet]. 2018; 6(3): 25-29. Disponible en: https://www.medigraphic. com/pdfs/conamed/con-2001/con013f.pdf

51. Kraus A, Cabral A, Villoro L. Los linderos de la ética. México: Siglo xxi; 2005. Ética y Biología; p. 109-132.

52. Rotondo M. Introducción a la bioética. Rev. urug. cardiol [Internet]. 2017; 32(3): 240-248. Disponible en: https://doi.org/10.29277/RUC/32.3.4

53. de Bennato MI. Los principios bioéticos en el proceso de toma de decisiones médicas. Nordeste. 2018; (12): 21-25. Disponible en: https://revistas.unne.edu.ar/index.php/nor/article/view/2990/2661

54. González R. El “paciente difícil” y profesionalismo médico. Rev. Cirugía [Internet]. 2019; 71(3). Disponible en: https://doi.org/10.4067/s2452-45492019000300270

55. Moreno J, Alvárez C, Wandel C, Lozano M. Glosario de términos y expresiones frecuentes de Bioética en la práctica de la Nutrición Clínica. Nutr. hosp [Internet]. 2010; 25(4): 543-548. Disponible: http:// scielo.isciii.es/scielo.php?script $=s c i$ arttext\&pi$\mathrm{d}=$ S0212-16112010000400004

56. Druml C, Ballmer P, Druml W, Oehmichen F. Guías de la ESPEN sobre aspectos éticos de la nutrición e hidratación artificial. Metabolismo y Nutrición Clínica. 2016; 98.

57. Cabeza de Vaca M, Aguilar M, Vizcaya M, Novalbos J, Lorenzo A, Valero J. Relevancia del soporte nutricional especializado en las declaraciones de voluntades anticipadas: experiencia de un centro hospitalario de especialidades. Nutr. Hosp [Internet]. 2017; 34(1): 6-14. Disponible: http://dx.doi.org/10.20960/nh.969 
58. Villares M, Manuel J. Hidratación y alimentación en los recién nacidos: adecuación del esfuerzo terapéutico. Cuadernos de Bioética [Internet]. 2015; 26(2): 1-13. Disponible en: http://aebioetica.org/revistas/2015/26/87/241.pdf

59. Moreno J, Galiano M. Consideraciones éticas sobre el papel de la nutrición parental domiciliaria. Persona y Bioética [Internet]. 2004; 8(23): 44-54. Disponible en: https://www.redalyc.org/pdf/832/83202306.pdf

60. Cañizo Ad. Nutrición en el paciente terminal. Punto de vista ético. Nutr. Hosp [Internet]. 2005; 20(2): 8892. Disponible en: http://www.nutricionhospitalaria. com/pdf/3530.pdf

61. Navas P. La relación médico-enfermo. Rev. S. And. Traum. y Ort .[Internet] 2019; 36(2): 6-7. Disponible en: http://revista.portalsato.es/index.php/Revista_ SATO/article/download/127/110 